\title{
Bronchoscopic Cryoextraction: A Novel Approach for the Removal of Massive Endobronchial Blood Clots Causing Acute Airway Obstruction
}

\author{
Bhaskar Bhardwaj MBBS (bhaskar_bhardwaj@hotmail.com) ${ }^{1}$ \\ Himanshu Bhardwaj MD (himanshu-bhardwaj@ouhsc.edu) ${ }^{2}$ \\ Houssein A. Youness MD (houssain-youniss@ouhsc.edu) ${ }^{2}$ \\ Ahmed Awab MD (ahmad-awab@ouhsc.edu) ${ }^{2}$
${ }^{1}$ Indira Gandhi Medical College, Department of Pulmonary Medicine and Tuberculosis, Shimla, Himachal Pradesh, India
${ }^{2}$ Pulmonary Medicine \& Critical Care, University of Oklahoma Health Sciences Center, Oklahoma City, OK USA

\begin{abstract}
Acute airway obstruction due to large blood clots is known to cause life threatening hypoxemic respiratory failure which can be challenging to diagnose and manage. Different bronchoscopic modalities like rigid bronchoscopy, forceps, snares and catheters can be used to extract these obstructing blood clots but each of these different methods have their own limitations. We describe a patient with iatrogenic endobronchial bleed with acute airway obstruction due to massive blood clot successfully managed using 'cryoextraction'. This technique has been described as the treatment of choice for this clinical situation and this case highlights the fact that this technique can save patients from more aggressive invasive procedures.
\end{abstract}

\section{Introduction}

Bronchoscopic cryoextraction using a cryoprobe is an infrequently used therapeutic modality for the removal of tracheobronchial tree foreign bodies, especially those containing sufficient water or freezable liquid (1). This technique uses a liquid cryogen or coolant (usually nitrous oxide, nitrogen, or carbon dioxide) which is delivered under pressure to a specially designed cryoprobe that can be passed through the working channel of the flexible bronchoscope (2). We present a case of acute life-threatening airway obstruction caused by large iatrogenic blood clots which was successfully managed using cryoextraction.

\section{Case Report}

A 54 years old male with history of renal transplant and chronic immune suppressive therapy was admitted to the intensive care unit with productive cough, fevers and dyspnea of 3 days duration. His initial vital signs showed blood pressure at 140/100 mm $\mathrm{Hg}$, pulse 110, respiratory rate at 36, temperature 102 degree Fahrenheit and initial oxygen saturation of $70 \%$ on supplemental nasal cannula oxygen at 4 liters/min. 
Physical examination revealed diffuse bronchial breath sounds in the right lower lung fields and chest radiograph showed consolidation in the right lower lobe. (Figure 1).

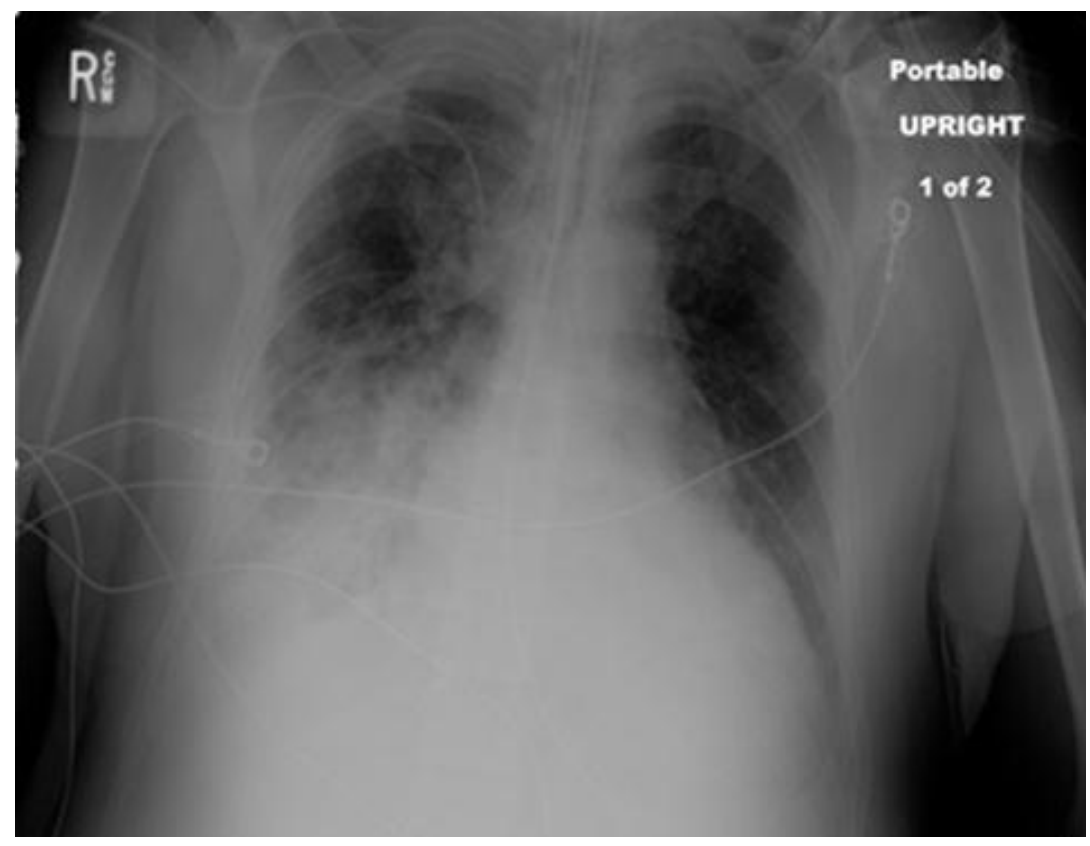

Figure 1. Pre BAL chest radiograph showing right lower lobe consolidation consistent with pneumonia.

Arterial blood gas analysis was consistent with partial pressure of oxygen (PaO2) at 40 $\mathrm{mm} \mathrm{Hg}$. Patient remained hypoxic despite supplemental oxygen and eventually required endotracheal intubation with mechanical ventilation due to hypoxic respiratory failure. Patient was also started on empiric antibiotic therapy with ceftriaxone and azithromycin for severe community acquired pneumonia requiring intensive care unit care. Unfortunately, patient's clinical condition deteriorated in next 48 hours despite continuous antibiotics. His oxygen requirements kept on escalating on mechanical ventilation besides continuous ongoing fever.

At this point, we decided to perform a bronchoscopy with a plan for bronchoalveolar lavage (BAL) given the high risk for atypical lung infections secondary to chronic immunosuppression in this patient. Airway examination during BAL showed extremely friable endobronchial mucosa with thick purulent secretions in the right lower lobe bronchi. Unfortunately, a massive endobronchial bleeding caused by an iatrogenic bronchial mucosal tear complicated the procedure. The most likely cause for this bleeding complication was bronchoscope induced mucosal trauma accentuated by vulnerability of the mucosal capillaries due to ongoing immunosuppression and pneumonia in this patient. BAL was terminated but patient became extremely hypoxic despite increasing fraction of inspired oxygen from initial $50 \%$ to $100 \%$. Acute rise in peak airway pressures to $56 \mathrm{~cm} \mathrm{H} 2 \mathrm{O}$ were also noted. An urgent repeat chest radiograph showed worsening of right lower lobe consolidation with new atelectasis suggestive of an acute airway obstruction (Figure 2). 


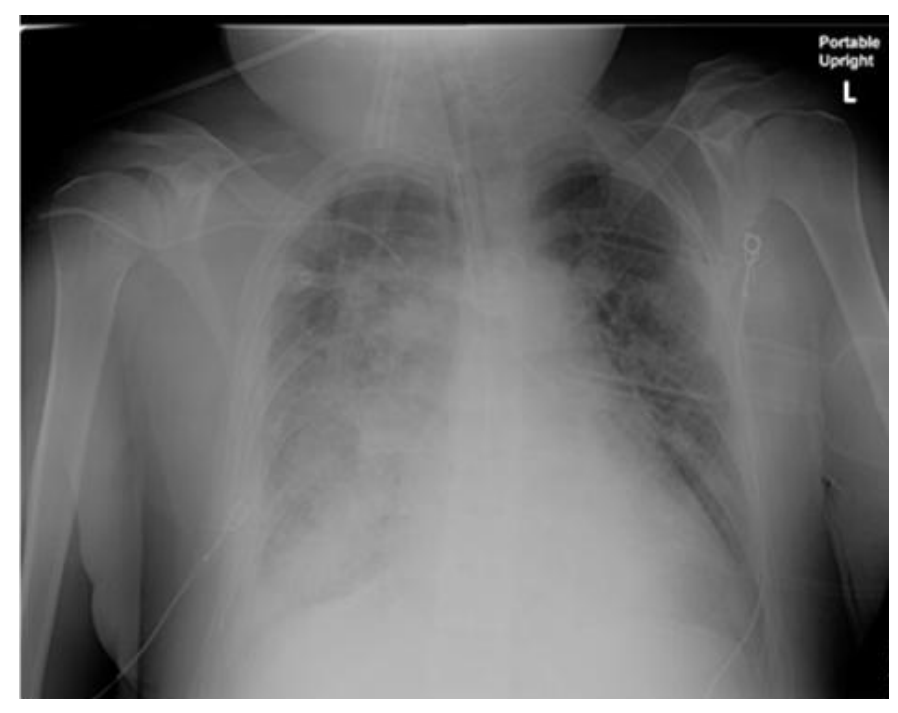

Figure 2. Post BAL chest radiograph showing acutely worse right lower lobe infiltrates, consistent with atelectasis and acute airway obstruction due to massive blood clot.

Repeat flexible bronchoscopic exam showed a massive blood clot extending from right main stem bronchus to lower bronchi obstructing the bronchial lumen almost completely. Removal of blood clot was felt to be necessary to improve the hypoxia. Initial attempts to suction the endobronchial clots through flexible bronchoscope and forceps extraction were unsuccessful due to extremely friable nature of the fresh blood clot. We decided to use cryoextraction to remove the endobronchial clot emergently.

A flexible cryoprobe (ERBE cryotherapy system - $1.9 \mathrm{~mm}$ size cryoprobe) was extended through the working channel of the bronchoscope into the bronchi, was applied to the clot \& frozen for 10 seconds. Frozen clot got firmly attached to the probe and it was successfully pulled out in one large piece (Figure 3 ).

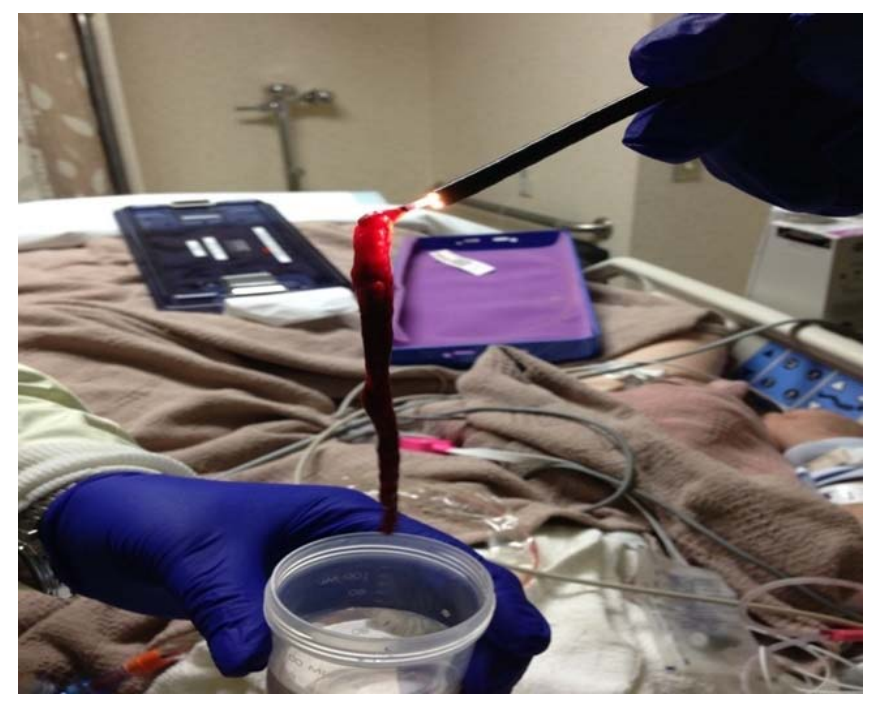

Figure 3. Massive blood clot extracted from airways, attached to the cryoprobe. 
This resulted in immediate improvement in patient's oxygenation. Patient remained on mechanical ventilation and a repeat bronchoscopic airway examination next day did not show any further bleeding. A non-bleeding mucosal tear in the right main bronchus was identified as the possible source of initial bleed. Patient eventually improved with continued treatment; he was successfully extubated after one week of mechanical ventilatory support. He was discharged home after total 2 weeks of hospitalization.

\section{Discussion}

Acute airway obstruction due to endobronchial blood clots is an unusual, but not a rare event which can develop in variety of clinical settings like various pulmonary infections, bronchial carcinoma, intrathoracic trauma etc. Some of the common interventions reported to cause acute airway bleeding and subsequent bronchial obstruction due to blood clots include: latrogenic mucosal damage from suction catheter manipulation, bronchoalveolar lavage, transbronchial biopsy and tracheostomy placement (Table1) (3).

\begin{tabular}{|c|}
\hline $\begin{array}{l}\text { Table1 : Important Causes of Airway Obstruction Arising from a } \\
\text { Endobronchial Blood Clot }\end{array}$ \\
\hline $\begin{array}{l}\text { 1.Pulmonary Infections : Tuberculosis, Pneumonia, Cavitory Fungal } \\
\text { Infections }\end{array}$ \\
\hline 2. Pulmonary Embolism \\
\hline 3. Pulmonary AV Malformations \\
\hline 4. Bronchiectasis \\
\hline $\begin{array}{l}\text { 5. Malignancy : Bronchogenic Carcinoma, Esopageal } \\
\text { Cancer, Lymphoma }\end{array}$ \\
\hline $\begin{array}{l}\text { 6. latrogenic Causes: } \\
\text { a. Endotracheal Intubation } \\
\text { b. Suction Catheter Manipulation } \\
\text { c. Bronchoalveolar Lavage } \\
\text { d. Transbronchial Biopsy } \\
\text { e. Percutaneous Tracheostomy } \\
\text { f. Thoracentesis \& Chest Tube Insertion }\end{array}$ \\
\hline 7. Other Causes : Trauma, Sarcoidosis, Epistaxis \\
\hline
\end{tabular}

Clinical consequences of the acute bronchial obstruction can range from minimal impact on respiratory function to life threatening ventilator failure. Pertinent physical examination findings in these patients include decreased or absent breath sounds with occasional inspiratory or expiratory wheezing heard over the affected lobe or lung. 
Among mechanically ventilated patients, acute rise in peak inspiratory pressure (above $60 \mathrm{~cm} \mathrm{H2O}$ ) with decreased tidal volume are some other notable findings. One unusual presentation of massive endobronchial bleeding in mechanically ventilated patients occurs when the clot adheres to the distal end of the endobronchial tube resulting in ball-valve type obstruction. In this situation, the clot acts as one-way valve allowing only the inspiratory flow into the lower respiratory tract but blocking the expiratory flow. This mechanism can result in unilateral or bilateral lung hyperexpansion, thus increasing the risk of tension pneumothorax. Urgent endobronchial tube exchange in this situation can be lifesaving (3). The extent of hypoxemia due to endobronchial blood clot obstruction depends on the site, degree of obstruction and underlying condition of the lungs (3). Typical imaging findings include lobar or segmental atelectasis or air column cut-off of the trachea and main stem bronchi. The diagnosis is confirmed by direct visualization of the clot through flexible bronchoscope. Initial efforts targeted at the removal of the blood clot involve suctioning and grasping forceps extraction of the clot through a flexible bronchoscope. However, these methods often prove unsuccessful due to the friable structure of the blood clots. Moreover, suctioning through the flexible bronchoscope could pose a risk of re-bleeding. Other management options include rigid bronchoscopy, Fogarty catheter dislodgment of the clot and sometimes the use of topical thrombolytic agents with partial dissolution of clot aiding in suction removal of the clot in piecemeal fashion. Rigid bronchoscopy with clot extraction was used to be the treatment to choice for the management of acute obstructing endobronchial blood clots but it requires general anesthesia and may not be as readily available as needed for these acutely sick patients $(4,5)$.

Cryoextraction using flexible cryoprobe is an underreported novel approach which can be successfully used in removal of large blood clots from the airways. One of the first descriptions of the use of cryoextraction in the removal of endobronchial blood clots was given by Mehta et al in one of their review about various interventions used in tracheobronchial foreign body extraction (6). This method allows freezing of the water component of the blood clots, leading to their removal in en-bloc. Additionally, freezing also has a hemostatic effect through vasoconstriction and rapid slowing of the circulation. Cryoextraction can also be used to extract mucus plugs and other foreign bodies containing some amount of freezable liquid. Under circumstances in which a foreign body does not have any or enough water content, one may consider spraying saline over the object and immediately freezing the foreign body; thus allowing successful cryoextraction. Freezing also leads to shrinking of the foreign objects, thereby easily separating them from inflamed mucosa and facilitating their removal. An additional advantage of this technique is the shorter learning curve needed to utilize the cryoprobe compared to the prolonged training required to master rigid bronchoscopy (7). One concern expressed about the cryoextraction of the massive endobronchial clots is that a large 'frozen clot' might be difficult to extract through the smaller sized endotracheal tubes and, if dislodged in that process, could lead to obstruction of the ET tube. 


\section{Conclusion}

Our case report illustrates the successful use of cryoextraction as a safe and cost effective tool which can be used in the quick removal of large airway clots causing symptomatic airway obstruction. This modality should be considered as the first line treatment in this clinical situation. ${ }^{1}$ Cryoextraction method can also spare patients from more invasive procedures like rigid bronchoscopy often used in these scenarios. ${ }^{6}$ In the absence of well-designed studies, this method must be objectively compared with other methods and more cases are needed to be analyzed in future studies.

\section{References}

1. Weerdt S, Noppen M, Remels $L$, et al. Successful removal of a massive endobronchial clot by means of cryotherapy. J Bronchol. 2005; 12:23-24. [CrossRef]

2. Rafanan AL, Mehta AC. Adult airway foreign body removal. What's new? Clin Chest Med 2001; 22:319. [CrossRef]

3. Arney KL, Judson MA, Sahn SA. Airway obstruction arising from blood clot: three reports and a review of the literature. Chest. 1999; 115(1):293-300. [CrossRef] [PubMed]

4. Schummer W, Schummer C. Hemorrhagic Tracheobronchial obstruction. J Bronchol. 2001; 8(3):236. [CrossRef]

5. Homasson JP, Vergnon JM .Cryotherapy to extract obstructing blood clots. J Bronchol 2002;9:158-9. [CrossRef]

6. Mehta AC, Rafanan A. Extraction of airway foreign body in adults. J Bronchol. 2001; 8:123-131. [CrossRef]

7. Rubio E, Gupta P, le S, Boyd M. Cryoextraction: a novel approach to remove aspirated chewing gum. Ann Thoracic Med. 2013; 8(1):58-59. [CrossRef] [PubMed]

Conflict of Interest disclosures: No financial or nonfinancial conflicts of interests exist for any of the involved authors. 